\title{
Combined direct visual and imaging guided percutaneous renal access using on shot tract dilation, a case series of novel methods of tract dilatation
}

Seyed Mohammad Kazem Aghamir ( $\nabla$ mkaghamir@tums.ac.ir)

Tehran University of Medical Sciences https://orcid.org/0000-0003-1611-0362

Fatemeh Khatami

Tehran University of Medical Sciences

Mohammad Rahimi

Tehran University of Medical Sciences

Hamidreza Zia

Tehran University of Medical Sciences

Research article

Keywords: PNL, tract Dilatation, nephrostomy

Posted Date: November 6th, 2019

DOl: https://doi.org/10.21203/rs.2.16840/v1

License: (c) (1) This work is licensed under a Creative Commons Attribution 4.0 International License.

Read Full License 


\section{Abstract}

Background: Percutaneous nephrolithotomy , (PCNL) is a common urological procedure for dilatation of access during one shot PNL.

Methods : Our research is over twenty one cases of one shot visually guided tract dilation PCNL who referred to Sina Hospital between 2017,2018 . They all underwent surgery using a specific 28 F dilatators with a customized central lumen which accepts a $4.5 \mathrm{~F}$ semi rigid ureteroscope to visually confirm the puncture of target calyx and passing a guide wire. This instrument complex was passed as a one shot dilator after withdrawal of the puncture needle.

Results: The mean duration time form insertion of a needle into target calyx until securing a guide -wire inside the collecting system (pelvis, ureter) and the mean time form needle withdrawal to successful dilation and sheath placement were 95 and 252 seconds respectively. There were no intraoperative or postoperative complications a result of this technique. There was no false passage or failed accesses .Total fluoroscopy time for percutaneous access was reduced considerably.

Conclusions: Use of modified ureteroscope kit during PNL, for visually confirmed Percutaneous nephrolithotomy , (PCNL) is feasible especially on complex calycealstones or pelvicalyceal Anatomies occasions.

\section{Background}

Percutaneous nephrilithotomy is the fundamental available endourological nephrolithiasis treatment (1, 2).Earning a secure percutaneous access to collecting system through fluoroscopic guidance and tract dilatation are crucial steps toward having a complete successful and safe procedure $(3,4)$. After successful puncture of the desired calyx, passage of the guide wire beyond the calyx into the pelvis and ureter provides the most secure position to proceed with access dilatation $(5,6)$.Yet many surgeon tend to change their with a more stiff on after the needle is removed to obtain a more rigid rail for introducing subsequent dilators, many have opted to use balloon dilators (7). It is advised to pass a guide wire into the ureter as safety wire or at least have one wire passed beyond the obstructed calyx or stone into the collecting system (in the upper pole calyx for instance). In order to prevent surgical complications and access failure due to dislodgement of the wire which could happen during several instrument changes for dilation of the tract and aggravated by kidney movement along breathing movements it is suggested to pass a guide wire into the ureter before starting dialation. The large stone bulk occupying the calyx the infundibulum, so it would impossible to pass the wire further down to renal pelvis or ureter which would result in a challenging tract dilation and higher rates of access failure due to wire dislodgement $(8,9)$. In our health center (Sina hospital,Tehran university of medical sciences) we have been using miniaturized PNL in both children and adults using ureteroscope and in few challenging cases we entered the calyx before dilation with the ureteroscope to disintegrate the stone and deploy a wire to proceed further with the dilation to full size. Revisions of this technique led to develop 
Amplatz dilator which could hold the ureteroscope inside and be used as a single shot dilator for percutaneous access. Feasibility of percutaneous access tract dilation especially in animal models is shown using a visual dilator system (10) and the human studies are required. Here we are presenting report of simulator experiences regarding visual dilation of the access tract as the first case series of combined direct visual and imaging guided percutaneous renal access using on shot tract dilation

\section{Methods}

Patients were recruited after completing the informed consent. Data on patients who had to undergo PNL and were referred to our center were gathered from 2017 to 2018 years due to ongoing clinical trials in end urology research group with ethical code of Tehran University of Medical Sciences (IR.TUMS.MEDICINE.REC.1398.237). The patients would routinely undergo abdominal CT scans urine culture and routine laboratory blood tests. PNL was carried out in prone position under general anesthesia by single surgery team. Retrograde ureteric catheterization and fluoroscopy helped in defining the calyceal anatomy. Puncture was performed with an 18 G PCN puncture needle with standard method. Free flow of urine/saline or ante grade contrast instillation through the needle would confirm successful entry and a $\mathrm{J}$ tip wire was passed through the needle into the collecting system in challenging cases in which the entire calyx of entry is filled up with the stone or due to certain infundibular anatomy and the wire could not be passed into the pelvis (figure 1). A pediatric ureteroscope (4.5-6 Fr by R.WOLF) irrigated by saline and preloaded with a specifically designed pencil shaped plastic dilator $(28 \mathrm{Fr})$ and sheath $(30 \mathrm{Fr}$ ) (figure 2), were used to pass the tract into the punctured calyx and maneuver another guide wire (0.035 inch) alongside he stone through the infundibulum under direct visual guidance and then fluoroscopic confirmation (figure 3).

Figure 1: Lower calyx puncture, guide wired could not be located beyond the infundibulum of the punctured calyx.

Figure 2: The new design of access dilation.

Figure 3: The progress of new design access dilation is monitored simultaneously through both endoscopic (a) and fluoroscopic monitors (b). 
Then this wire and ureteroscope itself was used as the rigid guide and the dilator was advanced gradually and visual confirmation of correct placement of the dilator and the access sheath inside the collecting system was done. In many instances the surgeon passed the whole dilator kit (ureteroscope plus the dilator) in one shot, keeping the ureteroscope aligned over the wire under direct vision and push the dilator forward at the same time with the other hand. All stage could be followed on the fluoroscope before insertion of the nephroscope. The time for access dilation was recorded from withdrawal of the needle sheath up to point that working sheath.

In these case a $30 \mathrm{~F}$ access sheath and customized $28 \mathrm{Fr}$ pencil shaped plastic dilator bearing a central lumen were loaded over a semi rigid ureteroscope were used as a visually guided dilator. The rest of the procedure was then carried out in the standard manner. Data analysis was done using SPSS (v16) software [Statistical procedures for social sciences; Chicago, Illinois, USA]. Chi-square, Fisher and Pearson regression tests were used and were considered statistically significant at $P$ values less than 0.05 .

\section{Results}

The mean duration form insertion of needle into target calyx until securing a guide-wire inside the collecting system (pelvis, ureter) was 95 seconds (84-107). Fluoroscopy time (total time required to obtain the access but not the whole operation) was 7-9.8 seconds. There were no intraoperative or postoperative complications as a result of this technique. One patient needed transfusion postoperatively. One patient needed extensive antibiotic therapy due to fever on the second post operative dey. The guide -wire could be successfully passed beyond the obstructed calyx into the ureter or father possible calyx in all cases. There were no failed accesses. Age and previous open surgery wer not related to the time and results $(p>0.05)$. Such technique significantly reduced the time of fluoroscopy needed during these cases.

Table 1-Summary of results

\begin{tabular}{ll} 
Variables & Results \\
\hline Mean age (years) & $45.7(16.76)$ \\
\hline Previous open surgery & $4(19.0 \%)$ \\
\hline Filed Access & 0 \\
\hline Mean fluoroscopy time (second) & 8.3 \\
\hline Mean operation time from puncture to sheath insertion (Seconds) & 252
\end{tabular}




\section{Discussion}

Earning a safe and properly placed percutaneous access into the renal collecting system is the basis of having an effective and safe PNL. Tract dilation is typically achieved by several passages of exchangeable flexible dilators of the Amplatz type, metallic telescoping dilators of the Alken type, or a solitary practice inflatable high pressure balloon $(8,9)$, Over time some features of the surgery techniques and the endoscopy's equipments have been progressed to boost the success rate of the PNL procedure. Now, balloon dilation can be the most novel safest one, though it has the drawback of quite high cost and high failure rate in cases of prior surgery or peri-renal fibrosis (11). Our novel technique improved dilatation to reach a prosperous process to allow safe dilatation and show the significance of those two steps leading to greater satisfaction rate between surgeons. Here we represent a single institution interventional study about the substitute method of under vision dilatation of access during one-shot PNL procedure, particularly in cases of unsuccessful fluoroscopic placements with uncertainty about existence of the guide wire in the system. In fact when the needle is proceeding towards the related target, some radiological sign may confirm its correct insertion and dilation and operative sheath placing are the last steps of the percutaneous tract creation.

A meta-analysis study by Dehong and his colleagues was over the four tact dilation methods for PCNL including Amplatz dilation (AD), metal telescopic dilation (MTD), balloon dilation (BD), and one-shot dilation (OSD) methods (12) and represented interesting data. His result presented that one shot dilatation can considerably reduce the duration of fluoroscopy and terminate the hemoglobin lessening in comparison with metal telescopic dilatation. Recently, several methods and substitute techniques have been presented in literature with the aim of increasing outcome and passing a perfectly positioned guidewire. Penbegul et al (13) planned an innovative disposable PNL set, cost-effective one - shot PNL set made of a single 30-F dilator, 30-F sheath, and 8-F polyurethane dilator, as use of a one - shot dilatation technique during PNL with successful results same as our study. Retrograde nephrostomy by Lawson's in 1980 (14) was reported as well. Furthermore, Grasso et al defined the flexible ureteroscopic control in difficult patients (15). Shi et al., stated that later than the renal infundibulum was distended by stimulated dieresis, the kidney was then punctured under the ultrasonographic guidance via the fornix of the target calyx (16). The functional channel was dilated by a distinct planned pencil-shaped facial dilator. It was shown by Javali et. al., that after positive access to target calyx, guidewire was handed through the outer dilator sheath under fluoroscopic guidance into the calyx (17). Semi-rigid ureteroscope was passed percutaneously during the puncture wound between the two guidewires, watching its secure progress using both the endoscopic and the fluoroscopic monitors, till the semi rigid ureteroscope go to the target calyx and the stone can be observed. Then the third guidewire was passed through the operational channel of the semi rigid ureteroscope, maneuvered by the side of the stone, through the infundibulum, into the pelvis and ureter. After that first two guidewires were then detached and usual tract dilatation was then carried out over the guidewire that was positioned into the ureter, following withdrawing the semi rigid ureteroscope. The rest of the process was then done in the usual manner. This method was done over 85 patients and in 82 of them the passing of guidewire in to the ureter was successful with mean time of duration equal to 95 seconds (from ureteroscope to ureter entrance)(17). 
In our novel method the usage of ureteroscope was only done by as the accessory method for guidewire conducting, so ureteroscopy and dilatation was done simultaneously. Another special safety feature of described visually confirmed tract dilation (VC-PNL) is the ability to visualize the different tissues while the dilator is passing through them and minimizing the risk for invertent injury to adjacent organs such as colon, etc.

Beside we could state that using this technique might reduce might reduce radiation exposure significantly in such difficult cases which usually necessitate multiple passages for obtaining a safe tract.

\section{Conclusions}

Our technique is feasible and easy to learn and contrary to balloon dilation, it does not impose any extra instrument cost to the expenses. The present study has several limitations as it was included relatively few patients and was not include a control group. Furthermore, no complications in relation to our technique were observed, demonstrating that it is safe to instrument a newly created procedure for better dilatation. Use of the ureteroscope loaded with the dilator and sheath during PNL, Seems to be a feasible and safe technique for dilatation of access tract during one shot PNL.

\section{Declarations}

\section{Abbreviations:}

PCNL: Per coetaneous nephrolithotomy

Ethics approval and consent to participate: The study was run under the supervision of with Tehran University of Medical Sciences Ethical Committee with ethical code (IR.TUMS.MEDICINE.REC.1398.237).

Consent for publication: As our manuscript does not contain any individual persons' data, so consent for publication is not applicable in this section.

Availability of data and materials: Data will be available on request

Competing interests: All authors clam that there is no competing interest.

Funding: There is no funding.

\section{Authors' contributions}

SMKA is principal investigator who suggests the main idea of this research, FKH is the writer of the text, $\mathrm{MR}$ and $\mathrm{HZ}$ are surgeon who run this project over the SMKA supervision.

Acknowledgements: Special thanks to Urology Research Center (URC), Tehran University of Medical Sciences, Tehran, Iran. 


\section{References}

1. Aghamir S, Hosseini S, Gooran S. Totally tubeless percutaneous nephrolithotomy. Journal of endourology. 2004;18(7):647-8.

2. Aghamir SMK, Salavati A, Aloosh M, Farahmand H, Meysamie A, Pourmand G. Feasibility of totally tubeless percutaneous nephrolithotomy under the age of 14 years: a randomized clinical trial. Journal of endourology. 2012;26(6):621-4.

3. Aghamir SMK, Modaresi SS, Aloosh M, Tajik A. Totally tubeless percutaneous nephrolithotomy for upper pole renal stone using subcostal access. Journal of endourology. 2011;25(4):583-6.

4. Aghamir S, Modaresi SS, Aloosh M, Farahmand H, Hosseini SH, Meysamie A. Which is access suitable for a solitary upper pole renal stone? A possible novel criterion. Minerva Urol Nefrol. 2012;64(1):1-6.

5. Eryildirim B, Tuncer M, Camur E, Ustun F, Tarhan F, Sarica K. Renal access in PNL under sonographic guidance: Do we really need to insert an open end ureteral catheter in dilated renal systems? A prospective randomized study. Archivio Italiano di Urologia e Andrologia. 2017;89(3):226-31.

6. Yang B, Li J, Hu W, Huang X, Wang X. Standard-tract percutaneous nephrolithotomy accessed by two-step dilation for 3052 patients. Beijing da xue xue bao Yi xue ban= Journal of Peking University Health sciences. 2010;42(4):447-50.

7. Sharma GR, Maheshwari PN, Sharma AG, Maheshwari RP, Heda RS, Maheshwari SP. Fluoroscopy guided percutaneous renal access in prone position. World Journal of Clinical Cases: WJCC. 2015;3(3):245.

8. Bryniarski P, Stelmach P, Taborowski P, Rajwa P, Adamkiewicz M, Życzkowski M, et al. Percutaneous nephrolithotomy with amplatz and alken dilators: an eight-year single tertiary care centre experience. Medical science monitor: international medical journal of experimental and clinical research. 2016;22:4918.

9. Özçift B, Bal K, Dinçel Ç. A comparison of balloon and amplatz dilators in percutaneous nephrolithotomy: a retrospective evaluation. Turkish journal of urology. 2013;39(4):226.

10. Shah AK, Xu K, Liu H, Lin T, Xie K, Huang H, et al. The "visual dilator system": initial experimental evaluation of an optical tract dilation technique in percutaneous nephrolithotomy. Journal of endourology. 2013;27(7):908-13.

11. Nalbant I, Karakoyunlu AN, Yesil S, Ekici M, Zengin K, Ozturk U, et al. Comparison of dilation methods in percutaneous nephrolithotomy: which one is more successful? Journal of Laparoendoscopic \& Advanced Surgical Techniques. 2016;26(6):478-82. 
12. Dehong C, Liangren L, Huawei L, Qiang W. A comparison among four tract dilation methods of percutaneous nephrolithotomy: a systematic review and meta-analysis. Urolithiasis. 2013;41(6):52330.

13. Penbegul N, Dede O, Daggulli M, Hatipoglu NK, Bozkurt Y. A novel percutaneous nephrolithotomy (PCNL) set: The 'Economical One-shot PCNL Set'(Ecoset). Arab journal of urology. 2017;15(3):199203.

14. Frattini A, Barbieri A, Salsi P, Sebastio N, Ferretti S, Bergamaschi E, et al. One shot: a novel method to dilate the nephrostomy access for percutaneous lithotripsy. Journal of endourology. 2001;15(9):91923.

15. Grasso M, Lang G, Taylor F. Flexible ureteroscopically assisted percutaneous renal access. Techniques in urology. 1995;1(1):39-43.

16. Shi Y, Liang H-g, Yang X, Hai B, Wang L, Xing Y-f, et al. Ultrasonography-guided percutaneous nephrolithotomy with Chinese one-shot tract dilation technique based on stimulated diuresis: A report of 67 cases. Journal of Huazhong University of Science and Technology [Medical Sciences]. 2016;36(6):881-6.

17. Javali T, Pathade A, Nagaraj H. A Novel method of ensuring safe and accurate dilatation during percutaneous nephrolithotomy. International braz j urol. 2015;41(5):1014-9.

\section{Figures}




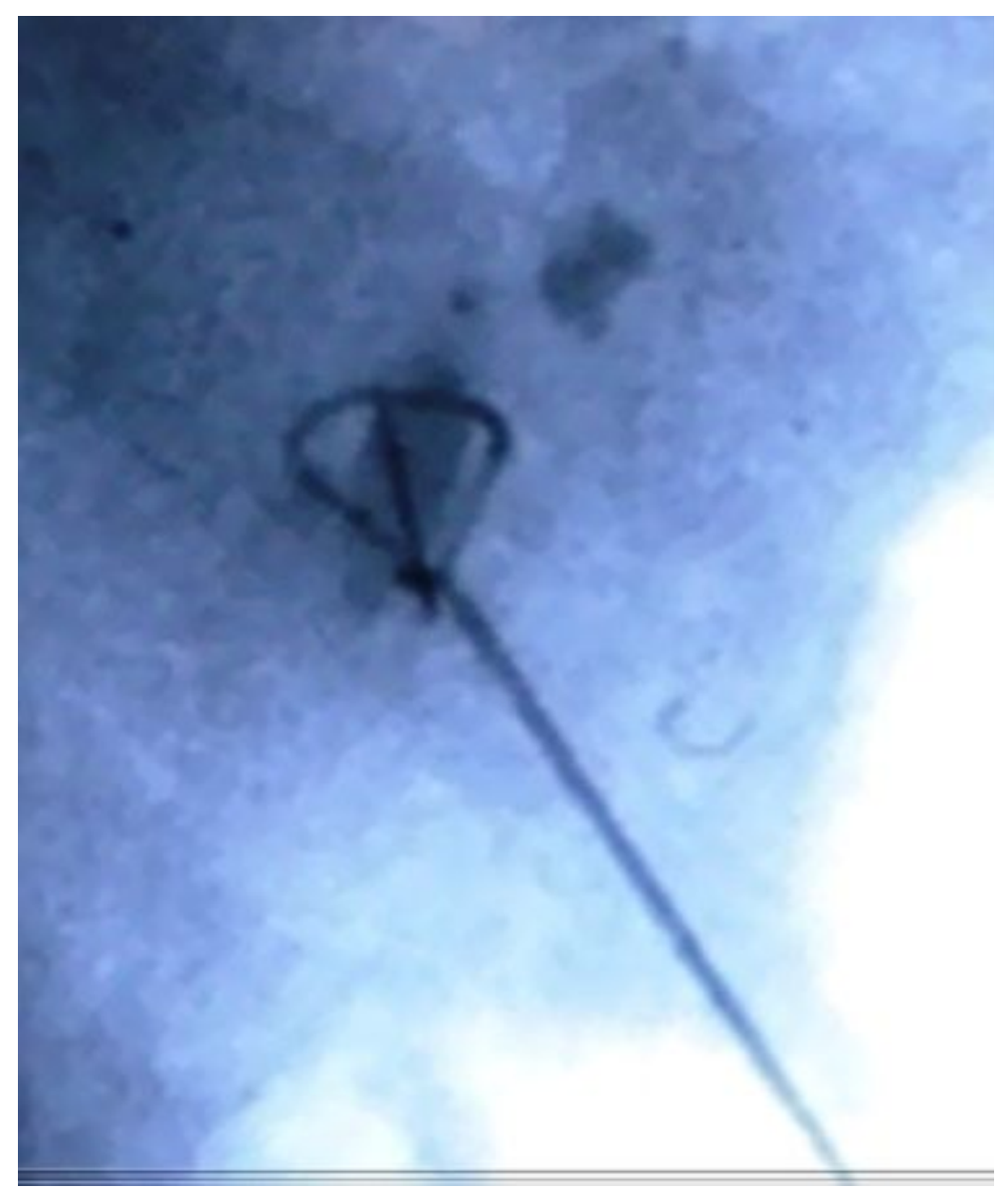

\section{Figure 1}

Lower calyx puncture, guide wired could not be located beyond the infundibulum of the punctured calyx. 

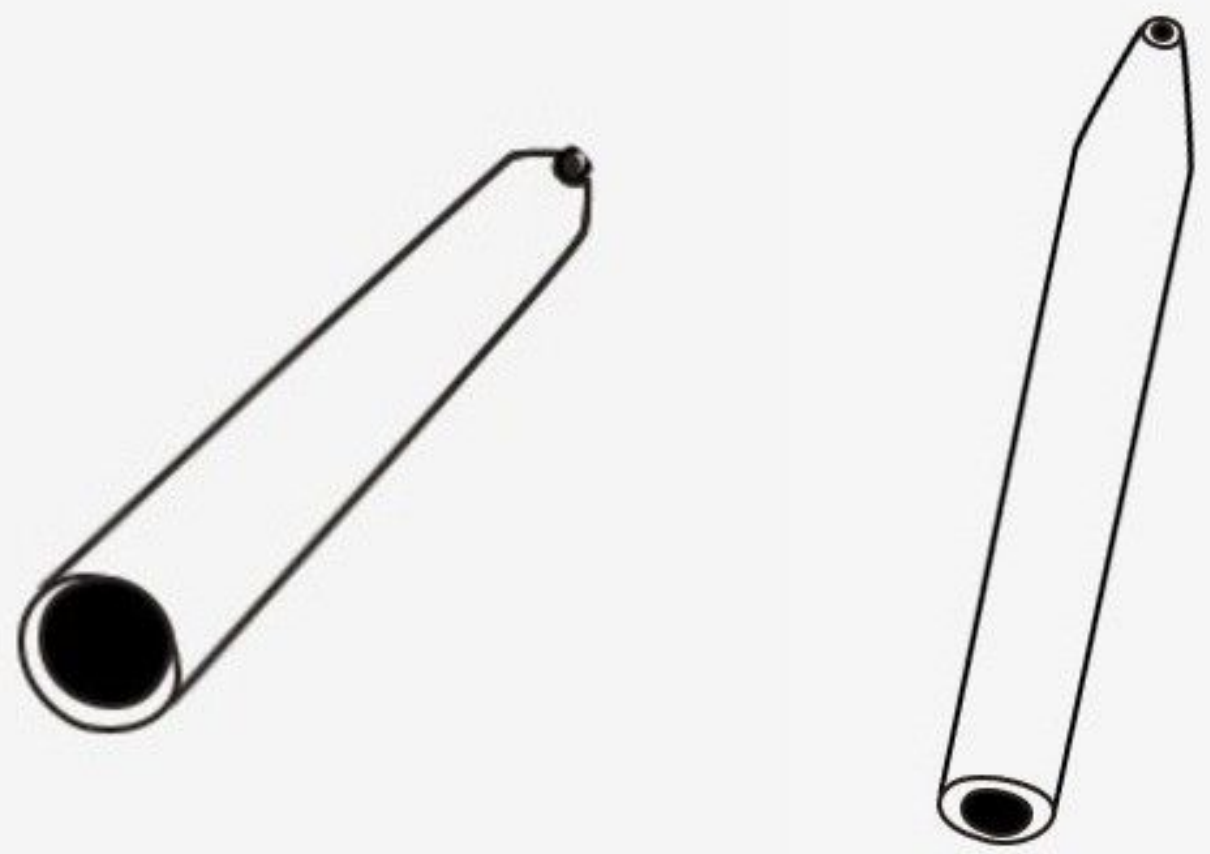

Figure 2

The new design of access dilation.

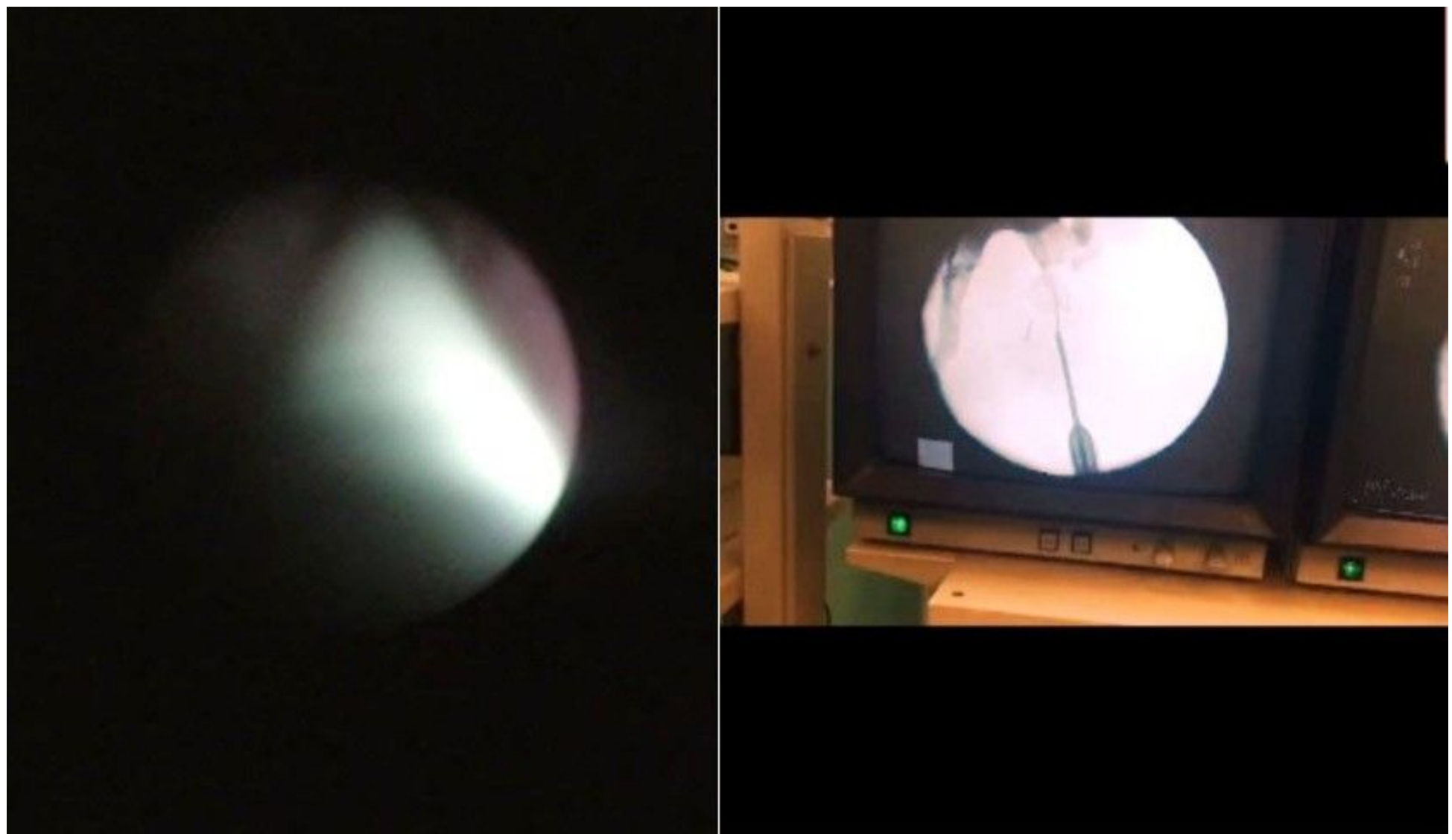




\section{Figure 3}

The progress of new design access dilation is monitored simultaneously through both endoscopic (a) and fluoroscopic monitors (b). 